\title{
Dalteparin improved chronic foot ulcers and reduced the number of amputations in diabetic peripheral arterial occlusive disease
}

Kalani M, Apelqvist J, Blomback $M$, et al. Effect of dalteparin on healing of chronic foot ulcers in diabetic patients with peripheral arterial occlusive disease: a prospective, randomized, double-blind, placebo-controlled study. Diabetes Care 2003;26:2575-80.

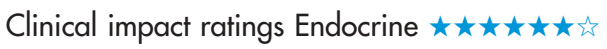

In diabetic patients with peripheral arterial occlusive disease (PAOD), is dalteparin more effective than placebo for improving chronic foot ulcers?

\section{METHODS}

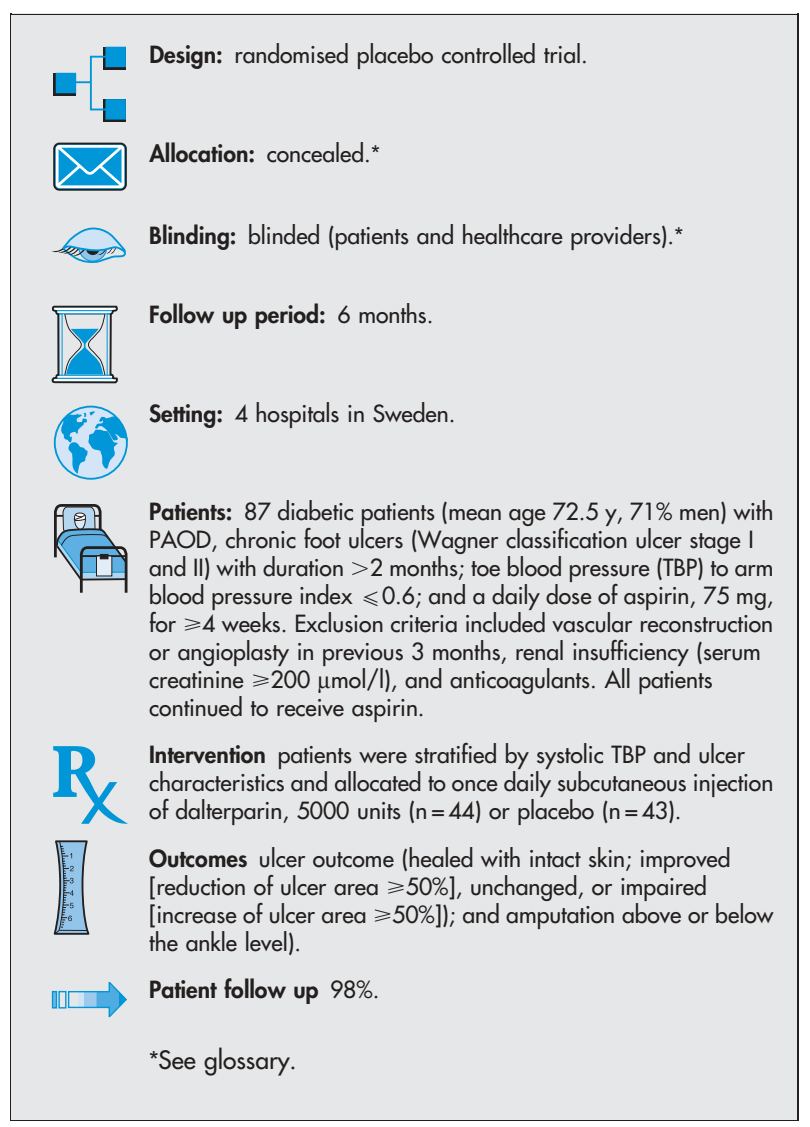

\section{MAIN RESULTS}

Analysis was by intention to treat. Patients who received dalteparin had greater improvement in ulcer outcome $(\mathrm{p}=0.042)$ and fewer amputations than those who received placebo (table).

\section{CONCLUSION}

In diabetic patients with peripheral arterial occlusive disease, dalteparin improved chronic foot ulcers and reduced the number of amputations.
Dalteparin $v$ placebo for chronic foot ulcers in diabetic patients with peripheral arterial occlusive disease at 6 months*

\begin{tabular}{lllll}
\hline Outcome & Dalteparin & Placebo & RRR $(95 \%$ CI) & NNT (CI) \\
\hline Amputation & $5 \%$ & $19 \%$ & $76 \%(6$ to 94$)$ & $7(4$ to 142$)$
\end{tabular}

*Abbreviations defined in glossary; RRR, NNT, and Cl calculated from data in article.

\section{Commentary}

he data in the study by Kalani et al show a trend toward increased healing, decreased ulcer size, and decreased amputation rate in the dalteparin group. Unfortunately, it is not known if patients underwent testing to exclude osteomyelitis prior to randomisation. In patients with diabetes, osteomyelitis is not a medically treatable condition (surgical debridement of all infected bone is essential), and wounds with osteomyelitis are unlikely to heal regardless of whether dalteparin is used. Therefore, the random inclusion of a few patients with occult osteomyelitis in the placebo group could skew the data and change the conclusions.

Clearly, it is bad to be a diabetic patient with a foot ulcer. Among patients in the placebo group, only $21 \%$ healed, $19 \%$ underwent amputation, and $59 \%$ still had an ulcer at final follow up. After the addition of dalteparin, the picture is still not pretty: only $33 \%$ of patients healed; $5 \%$ had an amputation; and a whopping $63 \%$ continued to have persistent ulceration.

Before dalteparin can be construed as producing a "benefit," we need to consider 2 questions: (1) does the presence of a small foot ulcer improve the quality of life relative to a bigger ulcer? and (2) how often is a patient better off with an amputation and a good prosthesis compared with a persistent (or even healed) ulcer on an ischaemic, poorly functioning marginal limb?

If these data are correct, treating 100 patients with diabetic foot ulcer with dalteparin will heal roughly 12 additional patients and prevent 14 amputations. At our institution, the lowest bulk purchase price for 5000 units of Fragmin is US $\$ 33$ per syringe. If the average patient requires 6 months of treatment, the cost of therapy would be approximately US $\$ 6000$ per patient, or $\$ 25000$ per ulcer healed or amputation avoided. Is it worth it? I don't know. This may be a social rather than a medical decision, but I agree with the authors' assessment that these data justify a larger, more definitive study.

Thom W Rooke, MD, FACC Mayo Clinic College of Medicine Rochester, Minnesota, USA
Sources of funding: Pharmacia Corporation; Swedish Heart-Lung Foundation; Stockholm County Council; R\&D Department of the Public Health and Medical Services Committee. 